\title{
Avaliação da Rotina Inversa $R 2 W$ na Estimação de Parâmetros de Transferência de Massa no Processo de Adsorção de Glicose e Frutose
}

A.L.J. BIHAIN ${ }^{1}$, Departamento de Engenharia Mecânica e Energia, DEMEC, Instituto Politécnico da Universidade do Estado do Rio de Janeiro, IPRJ - UERJ, 28625-530 Nova Friburgo, RJ, Brasil

L.D.T. CÂMARA2, Departamento de Engenharia Mecânica e Energia, DEMEC, Instituto Politécnico da Universidade do Estado do Rio de Janeiro, IPRJ UERJ, 28625-530 Nova Friburgo, RJ, Brasil

A.J. SILVA NETO ${ }^{3}$, Departamento de Engenharia Mecânica e Energia, DEMEC, Instituto Politécnico da Universidade do Estado do Rio de Janeiro, IPRJ UERJ, 28625-530 Nova Friburgo, RJ, Brasil

Resumo. As técnicas inversas são ferramentas muito utilizadas na determinação dos parâmetros envolvidos na modelagem de processos industriais. Neste trabalho o método Random Restricted Window (R2W) é empregado na estimação dos parâmetros de transferência de massa envolvidos na cromatografia da separação da glicose e frutose a partir do suco de caju. O R2W é um método estocástico simples, que analisa a função custo a partir de estimativas aleatórias pertencentes a um domínio definido para os parâmetros que se deseja ajustar, assumindo novas buscas aleatórias através de uma janela de domínio restrito próxima aos melhores candidatos à solução do problema de interesse. Na modelagem do processo cromatográfico foi utilizada uma nova abordagem fenomenológica, chamada de "velocidade da frente de convecção", a qual mostrou potencial na caracterização da coluna cromatográfica. Nesta, a velocidade da fase móvel (líquida) é considerada o fator dominante no transporte das moléculas ao longo da coluna. O método $R 2 W$ mostrou-se eficaz na obtenção dos parâmetros do modelo fornecendo uma ótima concordância entre os resultados da simulação computacional e os dados experimentais.

Palavras-chave. Problema Inverso, Algoritmo R2W, Cromatografia.

\section{Introdução}

A estimativa dos parâmetros desconhecidos em um modelo matemático a partir de dados experimentais constitui um problema inverso e é um passo muito importante

\footnotetext{
${ }^{1}$ aluis@iprj.uerj.br;

${ }^{2}$ dcamara@iprj.uerj.br;

3 ajsneto@iprj.uerj.br;
} 
para uma melhor compreensão dos fenômenos envolvidos em muitas áreas de aplicação [18]. A abordagem inversa é uma técnica muito utilizada em diversas áreas da ciência, como na modelagem de processos industriais, processos cromatográficos, etc. [3]. Nos problemas de identificação de parâmetros de modelos teóricos em engenharia química, muitos autores tem utilizado ferramentas como o algoritmo de Luus-Jaakola[11], Levenberg-Marquardt [12] entre outros métodos.

A cromatografia é um método físico-químico de separação fundamentada na migração diferencial dos componentes de uma mistura, que ocorre devido a diferentes interações entre duas fases imiscíveis, a fase móvel e a fase estacionária [9].

Entre os processos cromatográficos existentes, o Leito Móvel Simulado (LMS) tem sido muito aplicado em misturas de difícil difícil separação devido à eficiência do mesmo. Em função da aplicabilidade do LMS, muitos estudos vem sendo desenvolvidos com diferentes abordagens [15, 17]. Na modelagem do fenômeno, em geral são considerados diversos fatores, entre eles, dispersão axial, transferência de massa entre o fluido e a partícula, difusão intrapartícula e as cinéticas de adsorção e dessorção.

Neste trabalho foi proposta uma nova abordagem fenomenológica chamada "velocidade da frente de convecção"para descrever o processo de transferência de massa na cromatografia de adsorção da glicose e frutose presentes no suco de caju. Para estimar os parâmetros de adsorção e dessorção a partir dos dados experimentais [1], uma rotina inversa de otimização simples foi utilizada, o método Random Restricted Window $(R 2 W)[3]$.

A rotina $R 2 W$ é baseada em uma busca aleatória, que tem o domínio de busca reduzido de acordo com o quadrado dos resíduos entre os valores calculados com a simulação computacional e os dados experimentais. O método $R 2 W$ possui algumas peculiaridades com relação ao Algoritmo Genético (GA) e ao método de LuusJaakola (LJ), que restringem a região de busca de acordo com as melhores soluções obtidas a partir de estimativas aleatórias.

A abordagem fenomenológica "velocidade da frente de convecção"mostrou-se efetiva na caracterização da coluna cromatográfica para a separação de glicose e frutose presentes no suco de caju, assim como o método $R 2 W$ foi eficiente na obtenção dos parâmetros do modelo.

\section{Modelagem}

\subsection{Problema Direto}

O Leito Móvel Simulado (LMS) é um processo contínuo de separação de moléculas por cromatografia, no qual a modelagem e simulação podem ser utilizadas para encontrar as melhores condições operacionais para a separação. Entretanto a caracterização da coluna cromatográfica é uma etapa prévia e importante ná determinação dos principais parâmetros utilizados em diferentes processos cromatográficos, assim como no LMS.

Em um processo cromatográfico por adsorção líquido-sólido, a transferência de massa baseia-se no gradiente de concentração de moléculas entre as fases sólida e líquida, onde as moléculas do eluente (fase líquida) são adsorvidas pela fase sólida 
ocupando os sítios ativos na superfície desta. O limitante do processo é a capacidade máxima de adsorção de moléculas pela fase sólida.

Para descrever a transferência de massa que ocorre no processo cromatográfico uma nova abordagem chamada "velocidade da frente de convecção"foi desenvolvida. Esta considera que a velocidade da fase líquida é o fator dominante no transporte das moléculas ao longo da coluna cromatográfica [5]. Portanto o primeiro passo assumido na discretização foi a velocidade da fase líquida, seguido da transferência de massa entre a fase líquida e sólida. A velocidade da fase líquida foi obtida através do fluxo volumétrico da fase móvel através do meio poroso,

$$
v=\frac{Q}{\varepsilon A_{T}}
$$

onde $v$ é a velocidade da fase líquida, $Q$ é a vazão volumétrica, $\varepsilon$ é a porosidade e $A_{T}$ é a área total da coluna.

Para o cálculo da transferência de massa, a coluna cromatográfica foi discretizada em volumes de controle de comprimento $\xi$ que se movem ao longo da coluna com a mesma velocidade do fluxo do eluente, conforme pode ser observado na figura 1.

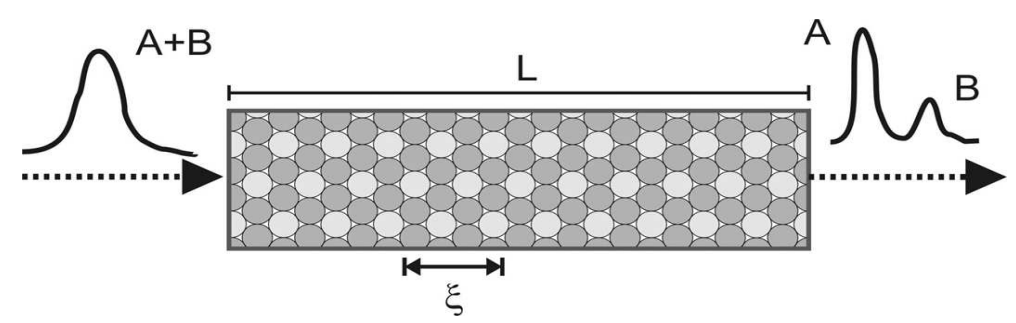

Figura 1: Representação da coluna cromatográfica.

Para caracterizar a cinética de transferência de massa do soluto entre a fase líquida e a fase sólida foi assumido o modelo global de cinética de transferência de massa,

$$
\left.\frac{d C}{d t}\right|_{\xi}=-k_{a d s} . C+k_{d e s} \cdot q
$$

onde, $C$ é a concentração na fase líquida, $q$ é a concentração na fase sólida, $t$ é o tempo, $k_{a d s}$ é a constante cinética de adsorção e $k_{d e s}$ é a constante cinética de dessorção.

Entre as principais características do modelo "velocidade da frente de convecção", podemos citar a facilidade de implementação e análise e ainda o mesmo necessita conhecer poucos dados operacionais do problema real. Porém ainda para conhecer as constantes cinéticas referentes às substâncias a serem separadas, se faz necessária a aplicação de uma rotina inversa. 


\subsection{Problema Inverso}

A análise do problema inverso constitui uma área multidisciplinar de pesquisa, com um grande número de aplicações em diferentes campos da ciência $[10,13,16]$. As técnicas inversas são de grande importância no estudo da adsorção, para a obtenção dos parâmetros dos modelos usados para simular esse fenômeno. Muitos estudos dessa natureza podem ser encontrados com diferentes abordagens, como por exemplo a aplicação de métodos determinísticos [19, 20] e métodos evolutivos com procedimentos aleatórios [4, 14], os quais mostraram que a combinação da análise de erros com uma rotina inversa pode ser satisfatória para uma estimativa de parâmetros com boa precisão.

Neste trabalho os dados experimentais correspondentes à separação da glicose e frutose presente no suco de caju, realizada em uma unidade LMS composta por um conjunto de onze colunas cromatográficas em série [1], foram ajustados ao modelo do problema direto apresentado anteriormente.

Para a obtenção dos parâmetros do modelo, foi usado o método Random Restricted Window $(R 2 W)$ [3], que é considerado um método estocástico simples, o qual utiliza um algoritmo de busca com uma distribuição aleatória. A figura 2 mostra uma representação esquemática do algoritmo.

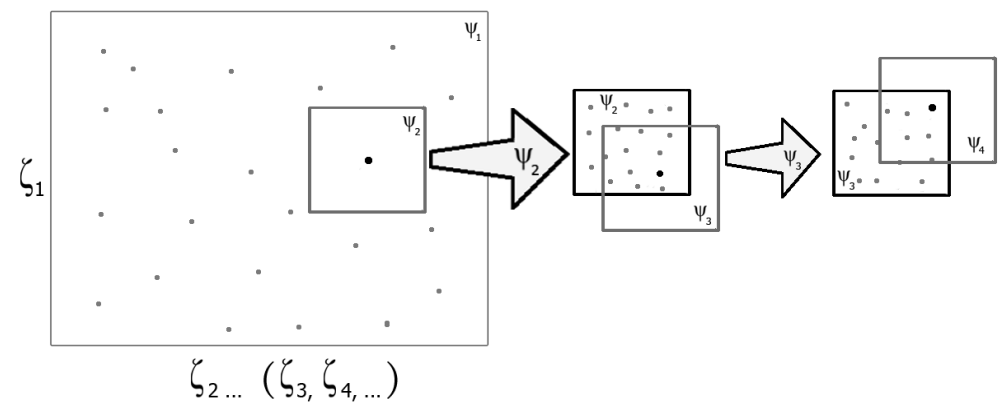

Figura 2: Representação esquemática do algoritmo R2W.

Na formulação do problema inverso de transferência de massa considerado nesse trabalho, busca-se minimizar uma função objetivo dado pela soma dos resíduos quadrados entre os valores experimentais e os valores calculados para a variável observável.

O algoritmo aleatório $R 2 W$, analisa a função objetivo a partir de estimativas aleatórias $(\zeta)$ pertencentes a um domínio definido $(Z)$ para os parâmetros que se deseja obter, escolhendo as melhores soluções nos intervalos de valores estimados para os parâmetros em que a função objetivo apresenta menor resíduo,

$$
\begin{gathered}
Z=f\left(\zeta_{1}, \zeta_{2}, \zeta_{3}, \ldots\right) \\
\zeta_{i}=\zeta_{i L}+R\left(\zeta_{i H}-\zeta_{i L}\right)
\end{gathered}
$$


onde $\zeta_{i L}$ e $\zeta_{i H}$ representam respectivamente o menor e o maior valor do parâmetro, ou seja, o intervalo ao qual as estimativas pertencem, sendo $R$ um número aleatório no intervalo $0 \leq R \leq 1$.

O procedimento representado na equação (2.4) é repetido para cada parâmetro $\zeta_{i}$, obtendo valores aleatórios pertencentes ao domínio definido conforme o número de sementes $S$ desejadas.

Após gerar todas as estimativas aleatórias pertencentes a um domínio para os parâmetros conforme o número de sementes definidos a priori, os resultados das simulações são comparados com os dados experimentais a fim de encontrar as melhores soluções. Para descobrir quais os valores para os parâmetros apresentam as melhores soluções é feita uma avaliação da soma dos resíduos quadrados $(Q)$,

$$
Q=\sum_{i=1}^{n p}\left(C_{\exp _{i}}-C_{\text {sim }_{i}}\right)^{2}
$$

onde $C_{\text {exp }}, C_{\text {sim }}$, e $n p$ correspondem respectivamente aos dados experimentais do processo de adsorção, os dados simulados pelo modelo e o número de dados experimentais.

Após a busca aleatória inicial $\Psi_{1}$, como pode ser visto na figura 2 , uma nova fase de buscas $\Psi_{2}$ é realizada em uma janela de domínio restrito, próxima às melhores solução obtidas na fase anterior, sendo o novo intervalo de busca definido pelo fator de restrição $(\delta)$ através das relações $(2.6)$ e (2.7), onde $\zeta_{i}^{*}$ corresponde à melhor solução para o parâmetro $i$ encontrado na fase anterior $\Psi_{1}$.

$$
\zeta_{L}=\zeta_{i}^{*}-\delta\left(\zeta_{i}^{*}\right)
$$

$$
\zeta_{H}=\zeta_{i}^{*}+\delta\left(\zeta_{i}^{*}\right)
$$

Na busca por uma solução melhor uma terceira fase $\Psi_{3}$ é realizada, repetindo o procedimento das etapas (2.6) e (2.7), realocando a janela de domínio restrito. Esse procedimento é mantido até que seja realizado um número de fases definido a priori. 


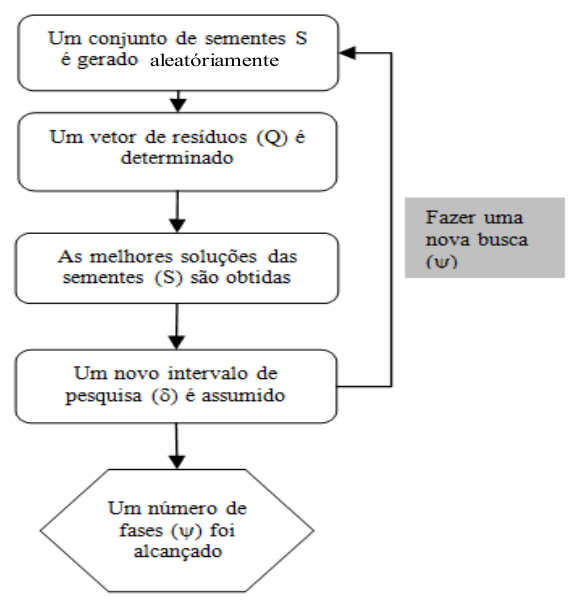

Figura 3: Diagrama representativo do algoritmo $R 2 W$.

O algoritmo $R 2 W$ representado no diagrama da figura 3 é um método evolucionário de busca. Pois o mesmo é um tipo de algoritmo evolutivo que constitui um método eficiente de otimização estocástica. Esse algoritmo realiza busca em uma população de possíveis soluções para o problema e utiliza uma estratégia de sobrevivência procurando as regiões em que melhor se adapta, ou seja, procura as regiões onde potencialmente estão as melhores soluções para poder refinar os resultados.

O algoritmo $R 2 W$ possui algumas características em comum com o algoritmo Luus-Jaakola e o Algoritmo Genético, pois ambos são métodos estocásticos de otimização. Entre estes o algoritmo $R 2 W$ possui uma semelhança maior com o LuusJaakola, pois ambos geram estimativas aleatórias dentro de um domínio de busca pré-estabelecido, entretanto no Luus-Jaakola, as restrições do domínio são realizadas ao longo das avaliações da função objetivo, enquanto no $R 2 W$ o domínio é reduzido somente na primeira fase, mantendo-se constante a área da região de busca nas fases posteriores.

\section{Resultados e Discussões}

Com a intenção de se avaliar a potencialidade da abordagem fenomenológica "velocidade da frente de convecção", e do algoritmo $R 2 W$ na solução do problema inverso, serão utilizados dados experimentais referentes a um pulso de concentração de uma amostra de $(300 \mathrm{ml})$ [1] de suco de caju injetado em uma coluna cromatográfica. Vale ressaltar ainda que a amostra do suco de caju é composta basicamente por uma mistura binária de glicose e frutose.

A critério de comparação, o algoritmo Luus-Jaakola também foi utilizado na solução do problema inverso. Os resíduos e os valores ótimos dos parâmetros de transferência de massa $k_{a d s}$ e $k_{\text {des }}$ da equação 2.2 obtidos pelos algoritmos $R 2 W$ e Luus-Jaakola podem ser observados na tabela 1. Para a execução do algoritmo $R 2 W$ foram usados $\delta=0,08, S=100$ e $\Psi=3$ e o domínio de pesquisa para os 
dois componentes da mistura foi $0 \leq k_{a d s} \leq 0.4$.

Tabela 1: Parâmetros ótimos de adsorção e dessorção para a glicose e frutose.

\begin{tabular}{|c|c|c|c|c|c|}
\hline Algoritmo & Componente & $k_{a d s}$ & $k_{\text {des }}$ & Resíduo $Q$ & Tempo Proc. em $s$ \\
\hline \multirow{2}{*}{ R2W } & Glicose & 0,01511 & 0,02810 & 6,18058 & 60,04 \\
\cline { 2 - 6 } & Frutose & 0,01454 & 0,01332 & 1,66883 & 56,41 \\
\hline \multirow{2}{*}{ Luus-Jaakola } & Glicose & 0,01749 & 0,03359 & 14,67762 & 99,99 \\
\cline { 2 - 6 } & Frutose & 0,01463 & 0,01353 & 1,74073 & 93,27 \\
\hline
\end{tabular}

Comparando na tabela 1 os resíduos que cada método apresentou, pode ser observado que para o caso estudado o $R 2 W$ apresentou um melhor desempenho, além de ter tempo de processamento inferior ao Luus-Jaakola. Para realizar estes ajustes, foram feitas 500 chamadas da função objetivo para cada algoritmo e para cada componente da mistura. A evolução do resíduo nas avaliações da função objetivo para cada um dos casos é apresentada na figura 4.

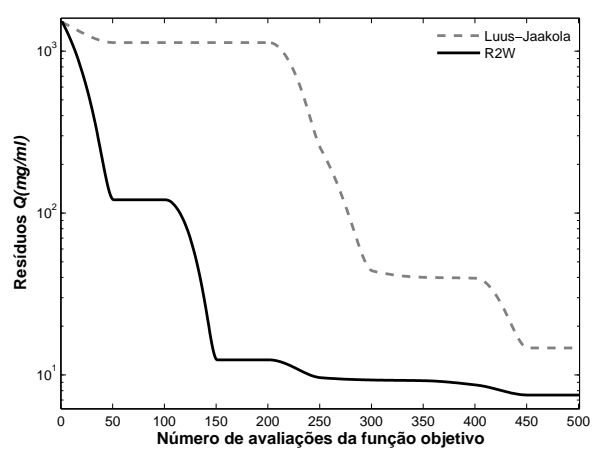

(a) Evolução da função objetivo no ajuste dos parâmetros para glicose.

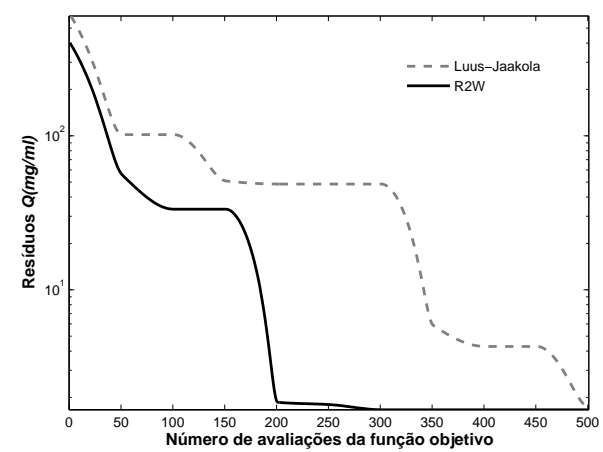

(b) Evolução da função objetivo no ajuste dos parâmetros para frutose.

Figura 4: Evolução da função objetivo através dos métodos Luus-Jaakola e R2W.

A partir dos resultados apresentados na figura 4, é possível afirmar que o algoritmo $R 2 W$ no caso estudado se aproxima mais rapidamente do valor mínimo da função objetivo e ainda ao final das avaliações chega a um valor absoluto de resíduo quadrado menor se comparado ao Luus-Jaakola.

A figura 5 mostra a correlação dos dados experimentais [1] com as simulações feitas a partir da abordagem fenomenológica do problema direto usando os parâmetros ajustados pelos métodos $R 2 W$ e Luus-Jaakola. Os resultados estão em termos da concentração do soluto na fase líquida em função do tempo (em minutos). 


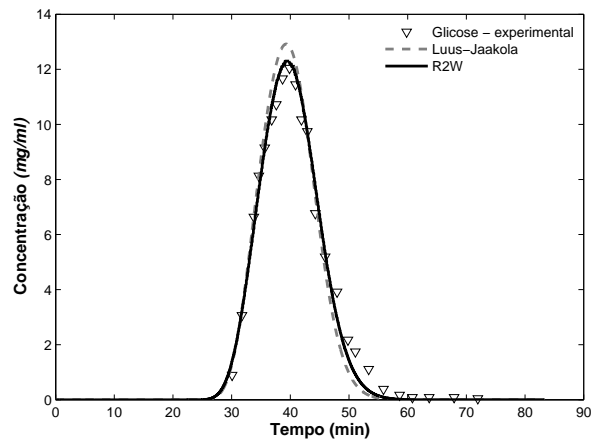

(a) Concentração glicose

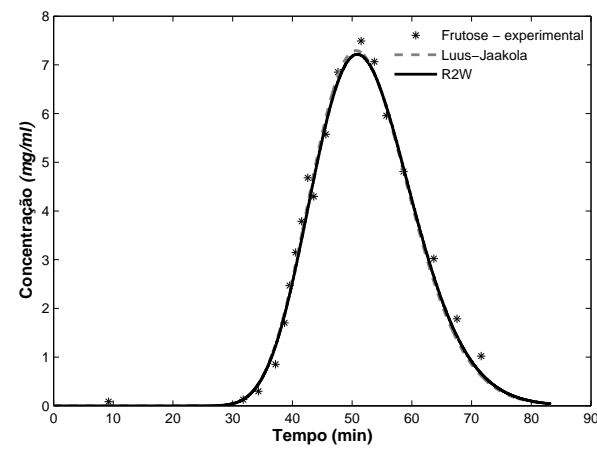

(b) Concentração frutose

Figura 5: Comparação dos dados experimentais da concentração da glicose 5(a) e frutose 5(b) no soluto [1] com os valores calculados.

Os resultados apresentados graficamente na figura 5 mostram o bom desempenho da abordagem "velocidade da frente de convecção"na representação da separação cromatográfica da glicose e da frutose presente no suco de caju, pois a simulação apresenta um perfil semelhante ao perfil dos dados experimentais. Assim como reafirma o bom desempenho do algoritmo R2W frente ao Luus-Jaakola para o problema abordado.

Para melhor entender o comportamento do algoritmo R2W e descobrir a melhor forma de operar o mesmo, uma avaliação dos parâmetros de controle do método foi realizada para cada componente da amostra de suco de caju. Para a análise de sensibilidade apresentada nas figuras 6 e 7 , foi utilizado o mesmo conjunto de sementes para a inicialização do gerador aleatório.

Na figura 6 estão representados os estudos das avaliações dos parâmetros de controle do método problema inverso aplicado no problema direto para a simulação da separação cromatográfica da glicose. Os resultados foram obtidos através de uma média de 3 simulações independentes do algoritmo $R 2 W$.

Na figura 6(a) é possível observar que existe uma relação inversamente proporcional entre o número de sementes e o resíduo, pois quando foi aumentado o número de sementes no algoritmo $R 2 W$, foram obtidas constantes para a glicose que resultaram em um resíduo menor entre a simulação e os dados experimentais. Como o método é estocástico, podemos dizer que quanto maior a quantidade de sementes, maior é a chance de obtermos bons resultados, ou seja, se encontrará constantes que vão resultar em menores resíduos.

Na figura 6(b) pode ser observado que se existe a necessidade de se encontrar uma solução refinada, o ideal é usar um fator de restrição menor, porém é necessário um número relativamente elevado de fases para se chegar ao resultado. Já com um fator de restrição maior existe a possibilidade de se chegar próximo à solução rapidamente, porém obter um resultado com boa precisão é pouco provável, pois o método ficará andando em torno da solução, pelo motivo de que este estará trabalhando com uma escala maior do que a da solução.

$\mathrm{Na}$ figura 7 podem ser observados os resultados dos estudos das avaliações dos 


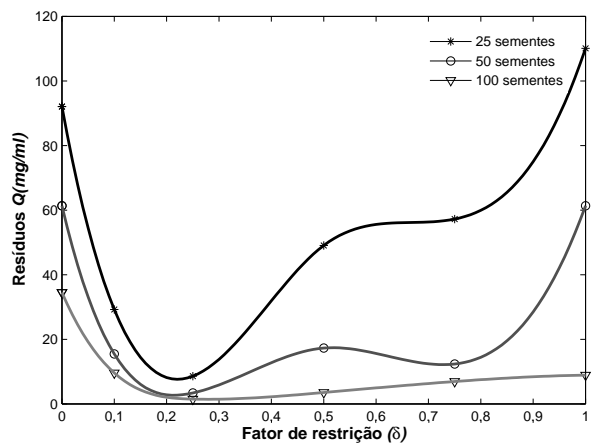

(a) Influência do número de sementes $(S)$ e do fator de restrição $(\delta)$

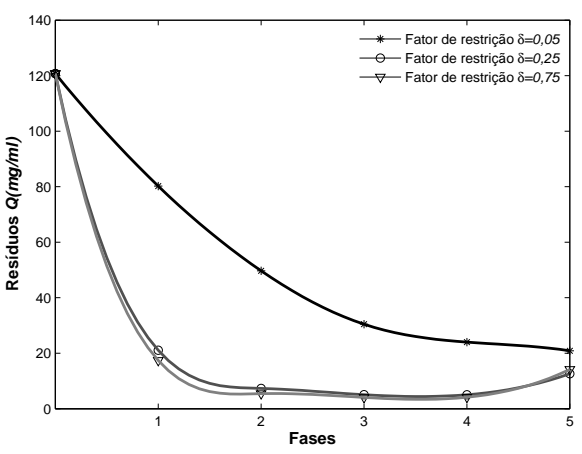

(b) Influência do fator de restrição $(\delta)$ e do número de fases

Figura 6: Avaliação dos parâmetros no método $R 2 W$, na busca das constantes de adsorção e dessorção da glicose.

parâmetros de controle do método $R 2 W$ para o ajuste dos parâmetros $k_{a d s}$ e $k_{\text {des }}$ do modelo da equação (2.2) para a adsorção da frutose adsorvida do suco de caju através do processo cromatográfico.

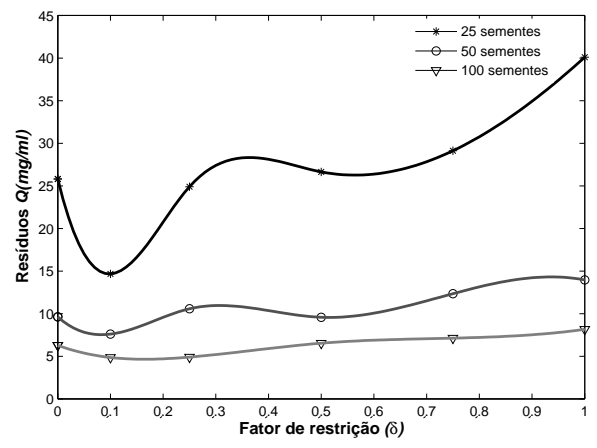

(a) Influência do número de sementes $(S)$ e do fator de restrição $(\delta)$

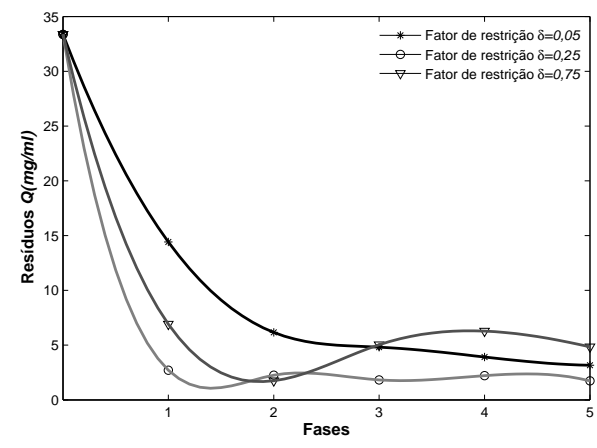

(b) Influência do fator de restrição ( $\delta$ ) e do número de fases

Figura 7: Avaliação dos parâmetros no método $R 2 W$, na busca das constantes de adsorção e dessorção da frutose.

Os resultados da figura 7(a) são semelhantes àqueles apresentados na figura 6(a), o que reafirma que aumentando o número de sementes, tem-se um resíduo menor, pois o aumento do número de sementes no $R 2 W$ faz com que aumente a densidade de estimativas no domínio levando a uma melhor solução.

Outra observação que pode ser feita a partir das figuras 7(a) e 6(a) é que usar um fator de restrição maior do que 0.5 não é recomendado, pois nesta faixa de valores o método começa a se distanciar da solução ótima.

A semelhança entre os resultados da figura 7(b) com 7(b) reafirma a hipótese 
que, quanto se deseja uma solução refinada a melhor estratégia a ser adotada é, escolher um fator de restrição relativamente pequeno e um elevado número de fases.

Na tabela 2 é apresentada uma comparação dos valores das constantes de equilíbrio da glicose $\left(k_{g l i}\right)$ e da frutose $\left(k_{f r u}\right)$ obtidas através de dois modelos com diferentes abordagens.

Tabela 2: Comparação das constantes de equilíbrio.

\begin{tabular}{|c|c|c|c|}
\hline & Modelo [1] & Este Trabalho R2W & Este Trabalho LJ \\
\hline \hline$k_{g l i}$ & 0,28 & 0,53 & 0,52 \\
\hline$k_{f r u}$ & 0,60 & 1,09 & 1,08 \\
\hline$\frac{k_{f r u}}{k_{g l i}}$ & 2,14 & 2,06 & 2,08 \\
\hline
\end{tabular}

Na abordagem usada em [1], considera-se os efeitos da transferência de massa no interior da partícula, tanto na fase líquida como na fase sólida, enquanto a utilizada neste trabalho considera que a velocidade da fase líquida é o fator dominante na transferência de massa.

É possível notar que apesar dos valores de $k_{g l i}$ e $k_{f r u}$ encontrados pelas duas abordagem serem diferentes, a relação entre os mesmos é semelhante, o que mostra que o modelo "velocidade da frente de convecção", apesar de considerar um número menor de fatores, é capaz de relacionar as concentrações dos componentes nas fases sólida e líquida de forma similar à modelagem clássica utilizada em [1].

\section{Conclusões}

A abordagem fenomenológica chamada "velocidade da frente de conveç̧ão"empregada neste trabalho para a simulação do processo de separação por adsorção da glicose e frutose presentes no suco de caju, possui potencial para ser usado na caracterização da coluna cromatográfica devido à simplicidade de implementação e à necessidade de um menor número de parâmetros em relação aos modelos clássicos.

O algoritmo $R 2 W$ usado na solução do problema inverso mostrou-se efetivo na obtenção das constantes de adsorção e dessorção tanto da glicose como da frutose, levando a boas soluções, as quais apresentaram um resíduo reduzido na correlação das simulações com os dados experimentais.

$\mathrm{O}$ algoritmo $R 2 W$ além de ser um método simples de implementar computacionalmente, mostrou um desempenho ligeiramente melhor e tempo de processamento inferior ao do algoritmo Luus-Jaakola na obtenção dos parâmetros de adsorção e dessorção estudados.

A avaliação dos parâmetros do algoritmo $R 2 W$ mostrou que a busca pela solução ideal é dependente do fator de restrição, número de fases e número de sementes aleatórias. A análise mostrou também que se o fator de restrição assumir valores maiores que 0,5 o algoritmo se distancia da solução ótima e ainda quando é aumentado o número de sementes aumenta a possibilidade de obter boas soluções com um 
número reduzido de fases.

Agradecimentos Os autores agradecem o apoio financeiro da FAPERJ, Fundação Carlos Chagas Filho de Amparo à Pesquisa do Estado do Rio de Janeiro, do CNPq, Conselho Nacional de Desenvolvimento Científico e Tecnológico, e da CAPES, Coordenação de Aperfeiçoamento de Pessoal de Nível Superior.

\begin{abstract}
Inverse techniques are tools widely used in the determination of parameters involved in the modelling of industrial processes. In the present work the method Restricted Random Window ( $R 2 W$ ) is employed to estimate the parameters of mass transfer involved in the chromatographic separation of glucose and fructose from cashew apple juice. The $R 2 W$ is a simple stochastic method, that evaluates the cost function for random estimates within the domain for the parameters to be adjusted, with new random searches performed within a restricted domain in the vicinity of the best solution candidates for the problem of interest. In the chromatographic process a new approach phenomenological is used, called "front velocity", which considers as the dominant factor in the molecules transfer in chromatographic columns the liquid phase velocity. The $R 2 W$ method has shown to be effective in the model parameters determination yielding a good agreement between the computational simulation and experimental data.
\end{abstract}

Keywords. Inverse Problem, Algorithm R2W, Chromatography.

\title{
Referências
}

[1] D.C.S. Azevedo, A. Rodrigues, Chromatography Applied to the Separation/Purification of Fructose from Cashew Apple Juice, Brazilian Journal of Chemical Engineering, 17 (2000), 4-7.

[2] L.D.T. Câmara, D.A.G. Aranda, Cheaction Kinetic Study of Biodiesel Production from Fatty Acids Esterification whith Ethanol,Industrial and Engineering Chemistry Research, Volume N 50 (2011), 2544-2547.

[3] L.D.T. Câmara, A.J. Silva Neto, Inverse Stochastic Characterization of Adsorption Systems by a Random Restricted Window ( $R 2 W$ ) Method, em "International Conference on Engineering Optimization (ENGOPT),", ENGOPT, 2008, Rio de Janeiro - RJ, 2008.

[4] L.D.T. Câmara, C.C. Santana, A.J. Silva Neto, Kinetic Modeling of Protein Adsorption With a Methodology of Error Analysis, Journal of Separation Science., Volume $\mathrm{N}^{\circ} 30$ (2007), 688-692.

[5] L.D.T. Câmara, Chromatographic Columns Characterization for SMB (Simulated Moving Bed) Separation of Glucose and Fructose, em "8th European Congress of Chemical Engineering,", Berlin, Germany, p 108-2011.

[6] M.C.M Castoldi, L.D.T. Câmara, D.A.G. Aranda, Kinetic Modeling of Sucrose Hydrogenation in the Production of Sorbitol and Mannitol with Ruthenium and Nickel-Raney catalysts, Reaction Kinetics and catalysts Letters, Volume $\mathrm{N}^{\circ} 98$ (2009), 83-89. 
[7] D.P. Costa, L.D.T. Câmara, M.Irizar-Mesa, O. Llanes-Santiago, D.C. Rodríguez, A.J. Silva Neto, Inverse and Direct Modeling Applied in the Estimation of Kinetic Parameters of BSA Adsorption,Ingenieria Electronica Automatica y Comunicaciones, Volume $\mathrm{N}^{\circ} 1$ (2009), 41-47.

[8] A.P.C. Cuco, A.J. Silva Neto, H.F. Campos Velho, F.L. Souza, Solution of an Inverse Problem with an Epidemic Genetic Algorithm and the Generalized, Extremal Optimization Algorithm, Inverse Problems in Science and Engineering., 17/3 (2009), 289-302.

[9] A.L.G. Degani, Q.B. Cass, P.C. Vieira, Cromatografia um breve ensaio, Quimica Nova Escola., $\mathrm{n}^{\circ} 7,1998$.

[10] F.L. de Souza, F.M. Ramos, P. Paglione, R.M. Girardi, A New Stochastic Algorithm for Design Optimization, AIAA Journal., 41/9 (2003), 1808-1818.

[11] F.S. Lobato, V. Steffen Jr., Algoritmo de Luus-Jaakola Aplicado a um Problema Inverso de Fermentação Batelada Alimentada, TEMA Tend. Mat. Apl. Comput. 9, No. 3 (2008), 417-426.

[12] J. Lugon Jr., A.J. Silva Neto, Estimativa de Isotermas de Adsorção Gás-Líquido Usando a Abordagem de Problema Inverso, TEMA Tend. Mat. Apl. Comput. 3, No. 2 (2002), 161-170.

[13] S. M. Al-Marzoug, R. J. W. Hodgson, Application of Luus-Jaakola optimization method to the desing of optical coatings, Proceedings of the 8th WSEAS International Conference on Evolutionary Computing. Vancouver, British Columbia, Canada, June 19-21, (2007), 229-234.

[14] M.Irizar-Mesa, O. Llanes-Santiago, F.H. Fernández, D.C. Rodríguez, A.J. Silva Neto and L.D.T. Câmara, An Approach to Parameters Estimation of a Chromatography Model Using a Clustering Genetic Algorithm Based Inverse Model, Soft Computing. 15 (2011), 963-973.

[15] A.K. Mostafazadeha, M. Sarshara, S. Javadiana, M.R. Zarefardc, Z.A. Haghighid, Separation of fructose and glucose from date syrup using resin chromatographic method: Experimental data and mathematical modeling, Separatio and Purification Technology. 79 (2011), 72-78.

[16] I. Poplewska, W. Piatkowski, D. Antos, Effect of temperature on competitive adsorption of the solute and the organic solvent in reverse-phase liquid chromatography, Journal of Chromatography A. 1103 (2006), 284-295.

[17] I.J. Silva Junior, M.A.G. Santos, V. Veredas, C.C. Santana, Experimental Determination of Chromatographic Separation Parameters of Ketamine Enantiomers on MCTA, Journal of Separation and Purification Technology. 43 (2005), $103-110$.

[18] A. Tarantola, Inverse Problem Theory and Methods for Model Parameter Estimation, SIAM, Paris, France, 2005. 
[19] J.F.V. Vasconcellos, A.J. Silva Neto, C.C. Santana, Estimativa do Coeficiente de Difusão e da Isoterma de Adsorção em Processo de Separação de Proteínas, em "4ํㅡㄹ Encontro de Modelagem Computacional,", Nova Friburgo - RJ, 2002.

[20] J.F.V. Vasconcellos, A.J. Silva Neto, C.C. Santana, An Inverse Mass Transfer Problem in Solid-Liquid Adsorption Systems, Inverse Problems in Engineering., 11/5 (2003), 391-408. 